\title{
VLDL cholesterol
}

National Diabetes Information Clearinghouse (NDIC)

\section{Definitions}

Cholesterol

Defined by National Diabetes Information Clearinghouse (NDIC)

Cardiovascular disease

Defined by National Diabetes Information Clearinghouse (NDIC)

\section{Source}

National Diabetes Information Clearinghouse (U.S.). (2009). The diabetes dictionary. [Bethesda, Md.]: U.S. Dept. of Health and Human Services, National Institutes of Health, National Institute of Diabetes and Digestive and Kidney Diseases, National Diabetes Information Clearinghouse.

Stands for very-low-density lipoprotein cholesterol. A form of cholesterol in the blood. High levels may be related to cardiovascular disease. 